\title{
Kirby-Bauer sensitivity test in postsurgical endophthalmitis
}

\author{
Raul Velez-Montoya*, Vidal F Soberon-Ventura, Juan M Elizondo-Camacho, Carl Sjoholm-Gomez de Liano, Maria Anna Martinez- \\ Castellanos, Guillermo Salcedo-Villanueva, Gerardo García-Aguirre
}

Retina Department, Asociación para Evitar le Ceguera en Mexico IAP, México City

\begin{abstract}
Purpose: To report the result of the Kirby-Bauer test in bacterial cultures from vitreous samples of patients with postsurgical endophthalmitis, in order to assess the bacterial response to first-line antibiotics like vancomycin and ceftazidime through time.

Methods: Retrospective, single center study. We reviewed the medical records of all post-surgical bacterial endophthalmitis cases, from 2009 to 2016 . We divided our population into two groups according to the date of diagnosis: group 1 (2009-2012) and group 2 (2013-2016). In each group, we noted the response of the grampositive bacteria to vancomycin and gram-negative bacteria to ceftazidime on the Kirby-Bauer test, as well as to the fourth-generation fluoroquinolones moxifloxacin and gatifloxacin. Nominal variables were assessed by Mann-Whitney test, with an alpha value of 0.05 for statistical significance. Chi-square test was applied to nominal variables as appropriate.
\end{abstract}

Results: Group 1: $46.2 \%$ of the gram-positive bacteria were sensitive to vancomycin and $83.4 \%$ of the gram-negative bacteria were sensitive to ceftazidime (moxifloxacin: $89.5 \%$, gatifloxacin: $68.4 \%$; $p<0.01$ ). Group 2: $47.4 \%$ of the gram-positive bacteria were sensitive to vancomycin and $66.7 \%$ of the gram-negative bacteria were sensitive to ceftazidime (moxifloxacin: $88 \%$, gatifloxacin: $84 \%$; $p<0.01$ )

Conclusion: The result found in this study suggest a possible change in the local sensitivity pattern that might have an impact on the treatment of postsurgical endophthalmitis. Moxifloxacin had higher levels of sensitivity and lower resistance on the Kirby-Bauer test than Vancomycin.

\section{Introduction}

Endophthalmitis is a rare, but potentially blinding condition, in where there is a contamination of the intraocular tissue by an exogenous or endogenous pathogen [1-3]. It is characterized by a severe inflammation, colonization, and destruction of the intraocular tissue $[1,2,4]$. Its incidence varies according to etiology and mechanisms of infection; however, the post-surgical cases are the most prevalent type [1]. Its incidence range from 0.03 to $0.3 \%$, depending on the primary surgical procedure from which endophthalmitis developed $[1,3,5,6]$. Coagulase negative staphylococci (Staphylococcus epidermidis) is the predominant pathogen-associated with cases of endophthalmitis due to cataract surgery $[1,7]$.

Visual outcome and organ preservation are strongly related to early diagnosis and prompt installment of treatment $[8,9]$. The imminent risk of permanent severe visual impairment forces immediate use of intravitreal broad-spectrum antibiotics; usually a combination of vancomycin and ceftazidime, with or without adjunctive oral moxifloxacin and oral or intravitreal steroids [8,10-13].

During the regular endophthalmitis hospital work-up, and before intravitreal antibiotics, vitreous samples (and sometimes aqueous samples as well) are drawn for microbiological studies and cultures $[1,9]$. The purpose is for the early identification of the causative pathogen and to assess its antibiotic susceptibility. The Kirby-Bauer disk diffusion method (KBT), is a laboratory technique that uses antibiotic-containing wafers or disks, placed in culture plates with bacteria isolated from the patient samples $[14,15]$. This method allows a fast assessment of the empirical treatment effect. and enables the clinician to properly select a more effective treatment in a case of low susceptibility [16]. The test is graded according to the area of bacterial inhibition surrounding the disk and the total area can be used to calculate the minimum inhibitory concentration [16].

Because the treatment outcome in endophthalmitis is closely tied to the susceptibility of the pathogen to the initial treatment; any change in the regional antibiotic susceptibility could potentially reduce the chances of a successful outcome [17]. Therefore, constant monitoring of the sensitivity profile of common bacterial strains is desirable. The aim of the current study is to review the results of all KBT performed on bacterial pathogens, isolated from patients with postsurgical endophthalmitis in the past 7 years (2009-2016), in order to describe the sensitivity to empirical treatment of endophthalmitis (vancomycin and ceftazidime) through time; in a single, large referral center, serving a population of 25 million inhabitants

\section{Methods}

This retrospective single center study was approved by the internal review board of the "Asociación para Evitar la Ceguera en Mexico, IAP" hospital ("Association for Blindness Prevention Mexico, IAP" hospital). The study was conducted according to the tenets of Helsinki and Good Clinical practices guidelines. All sensitive data were managed according to the Federal Law for Protection of Personal Data

Correspondence to: Raul Velez-Montoya, Vicente García Torres \#46. Col: San Lucas Coyoacán, México City, DF. 04030. Tel: +52.55.1084.1400; Fax: +52.55+1084.1404; E-mail: rvelezmx@yahoo.com

Key words: Kirby-Bauer; sensitivity; vancomycin; Staphylococcus epidermidis, endophthalmitis; outcomes

Received: January 02, 2018; Accepted: January 20, 2018; Published: January 25, 2018 
in Possession of Individuals (NOM-024-SSA3-2010) which is the local equivalent of the Health Insurance Portability and Accountability Act (HIPAA) rules of 1996. Due to the retrospective nature of the study, an informed consent was not needed at this time.

We reviewed all electronic medical records from the hospital's database from January 2009 to December 2016. The initial search was done, using the following code words and their combinations: endophthalmitis, bacterial, acute, intraocular, infection, intravitreal antibiotics, culture, endogenous and exogenous. The resultant medical records were then manually sorted and reviewed.

We included all medical records that met the following criteria: 1) Have a complete note of admission to the retina department, which includes a complete medical history and a comprehensive ophthalmological examination. 2) The case was immediately managed by a retina specialist or retina fellow, who administered intravitreal antibiotics and secured a vitreous and aqueous sample for microbiological examinations. 3) Have a complete microbiological report which includes data from the gram stains and cultures and the results of the standardized KBT. 4) There was evidence that the patient attended to all follow-up appointments, including a final assessment and discharge appointment. A case of postsurgical endophthalmitis was considered "resolved" when the visual acuity remained stable, with the absence of anterior chamber inflammation (clear cornea and no hypopyon), and without pain in the study eye for two weeks after the last intravitreal injection. After a successful isolation and growth in a culture media (Müeller-Hinton; NCCLS document M62-A7- Protocols for Evaluating Dehydrated Müeller-Hinton Agar), a bacterial strain was considered susceptible to an antibiotic, if at about 15 hours after disc exposure, the KBT reported an area of bacterial inhibition of at least 1 standard zone diameter in accordance with the recommendation of the Clinical Laboratory Standards Institute (CLSI document M100-S25 of 2015). For vancomycin, the KBT results were considered as sensible if the inhibition zone was $>12 \mathrm{~mm}$, Intermediate: $10-11 \mathrm{~mm}$ and Resistant: $<9 \mathrm{~mm}, 24$ hours after disc exposure.

We excluded all incomplete medical records and medical records in which the suspected or confirmed etiology was a fungus, cases with high suspicion of pseudo-endophthalmitis, or when a nonbiological agent was suspected to be responsible for the clinical presentation (toxic anterior segment syndrome). Only cases where the endophthalmitis was suspected to be secondary to a surgical procedure were included in the statistical analysis.

The reviewed files were divided according to the date of admission: group 1: files from Jan 2009 to Dec 2012 and group 2: files from Jan 2013 to Dec 2016. From each individual medical record, we recorded the date of admission, the suspected etiology of the endophthalmitis, relevant medical history, the best corrected visual acuity (BCVA) at the moment of admission/diagnosis, and at the date of discharge (endophthalmitis resolution), the number of days between the vitreous sample and the report of a positive culture result, the genus and species of the isolated pathogen grown in culture and the result of the KBT.

The BCVA was converted from Snellen letters to its logMAR equivalent for statistical evaluation. Visual acuities of count fingers (CF) equaled to $1.7 \operatorname{logMAR}$; hand movement (HM) to $2.0 \operatorname{logMAR}$; light perception (LP) to $2.3 \log$ MAR and no light perception (NLP) to 3.0 logMAR [18].

Statistical analysis was done using an excel spreadsheet (Excel 2010; Microsoft Corp., Redmond, WA) with and XLSTAT application v18.06 (Addinsoft, New York, NY). Data was presented as numbers and percentage for discrete variables and mean and standard deviation for continuous parameters. Change in BCVA and other nominal variables were assessed using a Mann-Whitney test, with an alpha value of less than 0.05 for statistical significance. The Chi-square test was applied to nominal variables as appropriate.

\section{Results}

A total of 92 medical records with a diagnosis of endophthalmitis were retrieved from the hospital electronic database (7 years survey). Sixty-two cases $(67.4 \%)$ were cases referred to the hospital from other ophthalmological clinics and private offices in the community. Thirty cases $(32.6 \%)$ were cases originated from our center. There were 49 females and 43 males. The mean age at presentation $54.3 \pm$ 19.7 years (range: 21 to 84 years). According to etiology, postsurgical endophthalmitis accounted for the majority, with precisely 44 cases. The 48 remaining cases were composed of 23 cases of post-traumatic endophthalmitis, 13 cases of endophthalmitis associated with corneal ulcers, 5 cases of endophthalmitis following intravitreal injections, 4 cases of endogenous endophthalmitis and 3 cases in which the source of endophthalmitis could not be confirmed.

From the 44 cases of postsurgical endophthalmitis, $47.7 \%$ were otherwise healthy, with no relevant medical history, at the moment of diagnosis. Twenty-two patients (50\%) had a history of diabetes; three of them with concomitant long-standing arterial hypertension and one more with hypercholesterolemia. Two patients were being treated for prostate cancer at the time of this study, and one more had a history of transurethral prostate resection due to benign prostatic hypertrophy. There was one case of postsurgical endophthalmitis in a patient with Marfan syndrome, and one patient who had a history of open-angle glaucoma. One patient (2\%) had a history of arterial hypertension with no other medical condition.

According to the date of diagnosis, 19 cases were allocated to group 1 (2009-2012) and 25 cases to group 2 (2013-2016). In group 1, endophthalmitis was diagnosed after a phacoemulsification surgery in 16 cases. Two more cases were diagnosed after pars plana vitrectomy and one case after a combined procedure of pars plana vitrectomy and scleral buckle. In thirteen cases a gram-positive bacteria were isolated from vitreous samples: 12 coagulase-negative staphylococci (9 in phacoemulsification, two in pars plana vitrectomy and one combined procedure) and 1 Enterococcus faecalis (phacoemulsification). In the remaining 6 cases, a gram-negative bacteria were isolated from the vitreous samples: 1 case of Moraxella nonliquefaciens, 2 cases of Serratia marcescens, 1 case of Proteus mirabilis, 1 case of Pseudomona aeruginosa and 1 case of Providencia stuartii. All of them were diagnosis after a phacoemulsification surgery. The mean number of days between vitreous sampling and a positive culture result was $6.6 \pm 4.9$ days. The mean number of days needed for case resolution was $15.8 \pm 4.3$ days. BCVA results and Change in BCVA from admission/baseline to case resolution are summarized in table. 1. According to the KBT, from the 13 gram-positive bacteria isolated in group $1,46.2 \%$ tested sensitive to vancomycin, while $53.8 \%$ tested resistant or intermediate (Resistant: 6 cases, Intermediate: 1 case). All cases but 1 (Pseudomona aeruginosa) of the gram-negative bacteria were sensitive to ceftazidime in the KBT (83.4\%). Regarding fourth generation fluoroquinolones, $89.5 \%$ of the 19 cases in group 1, were sensitive to moxifloxacin (1 case resistant, 1 case intermediate) and $68.4 \%$ to gatifloxacin respectively (4 cases resistant, 2 cases intermediate).

In group 2, endophthalmitis was diagnosis after a phacoemulsification surgery in 23 cases. Of the remaining two cases, 
Table 1. Mean change in visual acuity in group 1 and 2 . Overall, only patient in group 2 had a significant improvement in visual acuity after treatment ( $<<0001$ ). The wide range of visual acuity values and the existence of outliers could be the reason for this lack of significance. Subgroup analysis showed that patients with slightly better visual acuity $(<1.7$ logMAR) at baseline had better visual outcomes and achieved statistical significance. Those with worst visual acuity (>1.7logMAR) at baseline did not improve significantly in group 1 . However, patients with visual acuity ranging from hand movement to no light perception in group 2, had a significant improvement at the end of the study. BCVA: Best corrected visual acuity. logMAR: Logarithm of the minimum angle of resolution. SD: Standard Deviation. BL: Baseline. N: number of patients i the study group. *: Statistically significant value.

\begin{tabular}{|c|c|c|c|}
\hline & BCVA Baseline $(\log \mathrm{MAR} \pm \mathrm{SD})$ & Last BCVA $(\log$ MAR \pm SD) & $p$ value \\
\hline Group 1 & e & & \\
\hline \multirow[t]{2}{*}{ Overall $(N=19)$} & $1.94 \pm 0.5$ & $1.45 \pm 1.15$ & 0.1 \\
\hline & Range: 0.79 to 3.0 & Range: 0.097 to 3.0 & \\
\hline \multirow[t]{2}{*}{ BCVA at $\mathrm{BL}>1.7(N=12)$} & $2.26 \pm 0.39$ & $1.77 \pm 1.18$ & 0.4 \\
\hline & Range: 1.7 to 3.0 & Range: 0.17 to 3.0 & \\
\hline \multirow[t]{3}{*}{$\mathrm{BCVA}$ at $\mathrm{BL}<1.7(N=7)$} & $1.4 \pm 0.3$ & $0.9 \pm 0.9$ & $0.02 *$ \\
\hline & Range: 0.79 to 1.7 & Range: 0.09 to 3.0 & \\
\hline & BCVA Baseline $(\log \mathrm{MAR} \pm \mathrm{SD})$ & Last BCVA $(\log \mathrm{MAR} \pm \mathrm{SD})$ & $p$ value \\
\hline \multicolumn{4}{|l|}{ Group 2} \\
\hline \multirow[t]{2}{*}{ Overall $(\mathrm{N}=25)$} & $1.82 \pm 0.59$ & $1.04 \pm 084$ & $0.001 *$ \\
\hline & Range: 0.39 to 2.3 & Range: 0.09 to 2.3 & \\
\hline \multirow[t]{2}{*}{ BCVA at $\mathrm{BL}>1.7(N=17)$} & $2.1 \pm 0.15$ & $1.23 \pm 0.9$ & $0.005 *$ \\
\hline & Range: 2.0 to 2.3 & Range: 0.17 to 2.3 & \\
\hline \multirow[t]{2}{*}{ BCVA at $\mathrm{BL}<1.7(N=8)$} & $1.11 \pm 0.54$ & $0.63 \pm 0.5$ & $0.04 *$ \\
\hline & Range: 0.39 to 1.7 & Range: 0.09 to 1.7 & \\
\hline
\end{tabular}

1 was secondary to an extracapsular cataract extraction and the other secondary to a pars plana vitrectomy. In nineteen cases, a grampositive bacteria were isolated from vitreous samples: 14 coagulasenegative staphylococci (13 in phacoemulsifications, 1 in pars plana vitrectomy), 2 Staphylococcus saprophyticus (2 phacoemulsifications), 1 Enterococcus faecalis, 1 Micrococcus luteus (phacoemulsification) and 1 Staphylococcus aureus (phacoemulsification). In the remaining 6 cases, a gram-negative bacteria were isolated from each one of them: 1 case of Morganella morganii (extracapsular cataract extraction), 1 Bacteroides fragilis, 1 Chromobacterium violaceum, 1 Proteus mirabilis, 1 Haemophilus influenzae, 1 Moraxella catarrhalis (all of them after phacoemulsification). The mean number of days between vitreous sampling and a positive culture result was $6.4 \pm 2.7$ days. The mean number of days needed for case resolution was $20.4 \pm 8.2$ days. BCVA results and change in BCVA from admission/baseline to case resolution are summarized in table 1 . According to the KBT, from the nineteen cases of gram-positive bacteria isolated in group 2, $47.4 \%$ tested sensitive to vancomycin, while $52.3 \%$ tested resistant or intermediate (resistant: 9 cases, Intermediate: 1 case). Four cases of the gram-negative bacteria were sensitive to ceftazidime (66.7\%) and two (Proteus mirabilis and Haemophilus influenzae) were resistant (33.3\%). Regarding fourth generation fluoroquinolones, $88 \%$ and $84 \%$ of all cases tested sensitive to moxifloxacin and gatifloxacin respectively. Moxifloxacin reported $8 \%$ resistance and gatifloxacin $4 \%$ in the KBT.

Gram-positive bacteria showed no difference in resistance and sensitivity to vancomycin between group 1 and group $2(p=1.0)$. The KBT reported similar numbers of sensitive gram-negative bacteria to ceftazidime (5 out of 6 in group 1, and 4 out of 6 in group 2, $p=0.5$ ); Fourth generation fluoroquinolones displayed a higher level of sensitivity and low level of resistance among gram-positive and gram-negative bacteria in both groups. However, despite a slight improvement in the sensitivity level to gatifloxacin in group 2, there was no difference in resistance and sensitivity levels to moxifloxacin and gatifloxacin between group 1 and group $2(p=0.7$ and $p=0.07$ respectively).

In group 1, gram-positive bacteria had a significantly higher sensitivity to moxifloxacin than vancomycin $(\mathrm{p}<0.01)$ in the KBT. Despite the fact that gram-positive bacteria were more sensitive to gatifloxacin, there was no difference as against vancomycin $(p=0.3)$. In group 2, moxifloxacin and gatifloxacin had significantly higher sensitivity and lower resistance than vancomycin in gram-positive bacteria $(\mathrm{p}<001)$. There was no difference in resistance and sensitivity levels reported on the KBT of gram-negative bacteria between ceftazidime and fourth-generation fluoroquinolones, in group 1 and group $2(p=0.5)$

\section{Discussion}

The rise of highly resistant bacterial strains and multi drug-resistant strains is of high concern among all physicians regardless of specialty $[3,17,19]$. Various surveillance programs have reported a steadily increasing number of antibiotic resistance among gram-positive and gram-negative bacteria alike [17]. The Canadian Bacterial Surveillance Network reported increased resistance toward penicillin, trimethoprim sulfamethoxazole, macrolides and fluoroquinolones among 15,677 isolates of $S$. pneumoniae $[17,19]$. A high level of resistance toward erythromycin $(31 \%)$, and macrolides $(28 \%)$ was also reported for the same bacteria by the PROTEKT and TRUST studies $[17,20]$. The Canadian study also showed an emerging resistance toward the entire fluoroquinolone class of antibiotics, including fourth generation gatifloxacin and moxifloxacin [19].

Although the risk of acute bacterial endophthalmitis has remained low and relatively stable during the past two decades; the everincreasing number of ophthalmological surgical procedures, along with worldwide adoption of the intravitreal injections and the steady annual increase in the incidence of ocular trauma, has led to a rise in the total cases of bacterial endophthalmitis every year [21-24].

In addition to povidone-iodine instillation in the cul-de-sac [25]; several other countermeasures have been suggested as possible ways for endophthalmitis prophylaxis: The use of preoperative antibiotics as a way of eradicating sensitive conjunctival flora prior to surgery; the use of broad-spectrum antibiotics on the irrigating solutions and intracameral or subconjunctival injections of antibiotics after surgery, are some of such proposed measures $[3,7,25]$. Despite that there is no clear evidence supporting the benefit of this procedure [25], its practice has been highly adopted by ophthalmologists and continues to be used. Just in the United States alone, more than 75\% 
of ophthalmologist accepted using preoperative antibiotics, especially fluoroquinolones, as part of their routine preoperative practice [7]. However, complete sterilization of the conjunctiva prior to surgery is never achieved, and it has been speculated that these measures can contribute to the overall increase of bacterial antibiotic resistance, by selective elimination of susceptible strains and selecting genetically mutated strains for survival [26,27]. Recent large studies, including a report from the Diabetic Retinopathy Clinical Research Network, have found that pre-operative or pre-intravitreal injection antibiotics can be associated with an increased risk of developing an infection and accordingly advises against its routine usage [28-30]. It is believed that the introduction of the patient's own conjunctival and skin flora into the intraocular space, is an important risk factor for developing acute bacterial endophthalmitis [31,32], therefore, any change in the antibiotic susceptibility profile of the conjunctival normal flora may possibly induce an infection more difficult to treat; potentially with more negative outcome.

In this study, we analyzed the results of the KBT from postsurgical endophthalmitis cases within a period of 7 years. In both groups, the most frequently isolated bacteria from vitreous samples were CNS, with an overall predominance of gram-positive bacteria as the main bacteriological etiology. Our survey found that approximately $50 \%$ of all gram-positive bacteria isolated in cultures had an intermediate or decreased sensitivity to vancomycin. The trend is sustained through time, as shown by the results of group 1 and 2. Regardless of the low number of cases in each group, this observation deserves further analysis, especially because most studies assessing bacterial resistance to vancomycin agree that gram-positive bacteria remain highly sensitive to vancomycin $[7,22,23]$.

It is important to note that our gram-positive strains showed a better response (growth inhibition) to fourth generation fluoroquinolones, especially to Moxifloxacin. This observation contradicts the results from a very large study published by Miller et al from the Bascom Palmer Eye Institute [33]. In her study, Miller et al reported an emerging resistance to moxifloxacin and gatifloxacin, with $65 \%$ of the isolated CNS displaying cross-resistance to ciprofloxacin in cases of bacterial endophthalmitis. An increase in the Minimum inhibitory concentration $50\left(\mathrm{MIC}_{50}\right)$ and $\mathrm{MIC}_{90}$, as well as a decrease in the percentage of isolates inhibited by the $\mathrm{MIC}_{50}$, were consistently reported through time [33]. The CNS non-susceptibility rate for moxifloxacin of CNS rise from $22 \%$ in $1995-1999$ to $57 \%$ in $2010-2016$ [34]. The author argued that this trend may be the result of endophthalmitis prophylaxis patterns among ocular surgeons, In which fourth-generation fluoroquinolones, as well as older generations of fluoroquinolones like ciprofloxacin, are constantly being used $[33,34]$.

Vancomycin has been the first line antibiotic for the treatment of acute bacterial endophthalmitis since the Endophthalmitis Vitrectomy Study (EVS) reported excellent results in 1996 [35]. However, recent reports found a surge in vancomycin resistance, especially in Enterococcus (20\%) and Bacillus sp (4.17-32.3\%) [8,36-38]. Moreover, there are increasing number of reports linking the use of intravitreal or intracameral vancomycin with hemorrhagic occlusive retinal vasculitis, a potentially devastating condition caused by a delayed hypersensitivity reaction to vancomycin $[39,40]$. Another study from Mexico found an intermediate resistance to vancomycin $\left(\mathrm{MIC}_{90} 8\right.$ to $16 \mathrm{mg} / \mathrm{ml}$ ) in $28 \%$ of the studied CNS [41], which may help to partially explain the results of our study. Therefore, there is a compelling evidence of the need for a possible substitute to vancomycin as firstline treatment in cases of endophthalmitis. In our study, ceftazidime remains effective for the treatment of gram-negative endophthalmitis, with no significant difference in the level of sensitivity and resistance with fourth-generation fluoroquinolones. However, it is important to note that the number of cases of endophthalmitis due to gram-negative bacteria in our series were low. Therefore, this result should be taken with extreme consideration since it may not accurately depict the general population.

Finally, in addition to the retrospective nature and small sample, our study has limitations that we will like to address. The KBT is a test that requires trained personnel and might be subject to human appreciation and error. Since this is a retrospective study, we were not able to confirm our results by the determination of the $\mathrm{MIC}_{50}$ and $\mathrm{MIC}_{90}$ by laboratory methods. This is a significant drawback because the $\mathrm{MIC}_{50}$ and $\mathrm{MIC}_{90}$ are the current gold standard for assessing antibiotic resistance, which precludes us from making a definite recommendation. Nevertheless, we believe that our results are a potential reflection of a change in the local bacterial strains and their sensitivity to first-line antibiotics in cases of postsurgical endophthalmitis, which might have a huge impact on the clinical and functional outcome.

In summary, although, vancomycin and ceftazidime remain the first line treatment for acute bacterial postsurgical endophthalmitis, the results found in this study suggests a possible change in the regional bacterial susceptibility. Despite that the level of evidence found here is not enough for making a concrete recommendation regarding treatment, fourth-generation fluoroquinolones like moxifloxacin and gatifloxacin had higher levels of sensitivity and lower levels of resistance among gram-positive and gram-negative bacteria alike. A trend that was maintained through a 7 year period.

\section{Meeting presentation}

An early version of this manuscript will be presented at the Asociación Mexicana de Retina (AMR) annual meeting in Puerto Vallarta, México in October 2015 (Raul Velez-Montoya, MD.).

\section{References}

1. Vaziri K, Schwartz SG, Kishor K, Flynn HW Jr (2015) Endophthalmitis: state of the art Clin Ophthalmol 9: 95-108. [Crossref]

2. Reddy AK, Reddy RR, Paruvelli MR, Ambatipudi S, Rani A, et al. (2015) Susceptibility of bacterial isolates to vancomycin and ceftazidime from patients with endophthalmitis: Is there a need to change the empirical therapy in suspected bacterial endophthalmitis? Int Ophthalmol 35: 37-42. [Crossref]

3. Harper T, Miller D, Flynn HW, Jr. (2007) In vitro efficacy and pharmacodynamic indices for antibiotics against coagulase-negative staphylococcus endophthalmitis isolates. Ophthalmology 114: 871-875. [Crossref]

4. Liu K, Fang F, Li H (2015) Reliability of vitreous histological detection of pathogenic fungi in the diagnosis of fungal endophthalmitis. Eye (Lond) 29: 424-427. [Crossref]

5. Samant P, Ramugade S (2014) Successful use of intravitreal and systemic colistin in treating multidrug-resistant Pseudomonas aeruginosa postoperative endophthalmitis. Indian J Ophthalmol 62: 1167-1170. [Crossref]

6. Fileta JB, Scott IU, Flynn HW, Jr. (2014) Meta-analysis of infectious endophthalmitis after intravitreal injection of anti-vascular endothelial growth factor agents. Ophthalmic Surg Lasers Imaging Retina 45: 143-149. [Crossref]

7. Recchia FM, Busbee BG, Pearlman RB, Carvalho-Recchia CA, Ho AC (2005) Changing trends in the microbiologic aspects of postcataract endophthalmitis. Arch Ophthalmol 123: 341-346. [Crossref]

8. Hillier RJ, Arjmand P, Rebick G, Ostrowski M, Muni RH (2013) Post-traumatic vancomycin-resistant enterococcal endophthalmitis. $J$ Ophthalmic Inflamm Infect 3: 42. [Crossref]

9. Schwartz SG, Flynn HW Jr, Das T, Mieler WF (2016) Ocular Infection: Endophthalmitis Dev Ophthalmol 55: 176-188. [Crossref] 
10. Bertino JS Jr (2009) Impact of antibiotic resistance in the management of ocular infections: the role of current and future antibiotics. Clin Ophthalmol 3: 507-521. [Crossref]

11. Callegan MC, Gilmore MS, Gregory M, Ramadan RT, Wiskur BJ, et al. (2007) Bacterial endophthalmitis: therapeutic challenges and host-pathogen interactions. Prog Retin Eye Res 26: 189-203. [Crossref]

12. Bhagat N, Nagori S, Zarbin M (2011) Post-traumatic Infectious Endophthalmitis. Surv Ophthalmol 56: 214-251. [Crossref]

13. Comer GM, Miller JB, Schneider EW, Khan NW, Reed DM, et al. (2011) Intravitreal daptomycin: a safety and efficacy study. Retina 31: 1199-1206. [Crossref]

14. Ackerman BH, Dello Buono FA (1996) In vitro testing of antibiotics. Pharmacotherapy 16: 201-217. [Crossref]

15. Biemer JJ (1973) Antimicrobial susceptibility testing by the Kirby-Bauer disc diffusion method. Ann Clin Lab Sci 3: 135-140. [Crossref]

16. Wang N, Huang Q, Tan YW, Lin LP, Wu KL (2016) Bacterial spectrum and resistance patterns in corneal infections at a Tertiary Eye Care Center in South China. Int $J$ Ophthalmol 9: 384-389. [Crossref]

17. Karchmer AW (2004) Increased antibiotic resistance in respiratory tract pathogens: PROTEKT US--an update. Clin Infect Dis 39 Suppl 3: S142-150. [Crossref]

18. Lee JW, Lai JS, Yick DW, Tse RK (2010) Retrospective case series on the long-term visual and intraocular pressure outcomes of phacomorphic glaucoma. Eye (Lond) 24 : 1675-1680. [Crossref]

19. Patel SN, McGeer A, Melano R, Tyrrell GJ, Green K, et al. (2011) Susceptibility of Streptococcus pneumoniae to fluoroquinolones in Canada. Antimicrob Agents Chemother 55: 3703-3708. [Crossref]

20. Doern GV, Brown SD (2004) Antimicrobial susceptibility among community-acquired respiratory tract pathogens in the USA: data from PROTEKT US 2000-01. J Infect 48: 56-65. [Crossref]

21. Holz ER (2005) Reducing endophthalmitis risk factors: where we stand. $A m J$ Ophthalmol 139: 1097-1098. [Crossref]

22. Miller JJ, Scott IU, Flynn HW, Jr., Smiddy WE, Newton J, Miller D (2005) Acuteonset endophthalmitis after cataract surgery (2000-2004): incidence, clinical settings, and visual acuity outcomes after treatment. Am J Ophthalmol 139: 983-987. [Crossref]

23. Deramo VA, Lai JC, Winokur J, Luchs J, Udell IJ (2008) Visual outcome and bacterial sensitivity after methicillin-resistant Staphylococcus aureus-associated acute endophthalmitis. Am J Ophthalmol 145: 413-417. [Crossref]

24. Cedrone C, Ricci F, Regine F, Cerulli A, Palma S, Culasso F (2008) Nationwide incidence of endophthalmitis among the general population and the subjects at risk of endophthalmitis in Italy. Ophthalmic Epidemiol 15: 366-371. [Crossref]

25. Almeida DR, Miller D, Alfonso EC (2010) Anterior chamber and vitreous concordance in endophthalmitis: implications for prophylaxis. Arch Ophthalmol 128: 1136-1139. [Crossref]

26. Starr MB, Lally JM (1995) Antimicrobial prophylaxis for ophthalmic surgery. Surv Ophthalmol 39: 485-501. [Crossref]
27. Virk A, Steckelberg JM (2000) Clinical aspects of antimicrobial resistance. Mayo Clin Proc 75: 200-214. [Crossref]

28. Bhavsar AR, Torres K, Glassman AR, Jampol LM, Kinyoun JL, Diabetic Retinopathy Clinical Research N (2014) Evaluation of results 1 year following short-term use of ranibizumab for vitreous hemorrhage due to proliferative diabetic retinopathy. JAMA Ophthalmol 132: 889-890. [Crossref]

29. Chen RW, Rachitskaya A, Scott IU, Flynn HW Jr (2013) Is the use of topical antibiotics for intravitreal injections the standard of care or are we better off without antibiotics? JAMA Ophthalmol 131: 840-842. [Crossref]

30. Bhavsar AR, Ip MS, Glassman AR, Drcrnet, the SSG (2007) The risk of endophthalmitis following intravitreal triamcinolone injection in the DRCRnet and SCORE clinical trials. Am J Ophthalmol 144: 454-456. [Crossref]

31. Yin VT, Weisbrod DJ, Eng KT, Schwartz C, Kohly R, et al. (2013) Antibiotic resistance of ocular surface flora with repeated use of a topical antibiotic after intravitreal injection. JAMA Ophthalmol 131: 456-461. [Crossref]

32. Holland EJ, McDonald MB, Parekh JG, Sheppard JD (2014) Antibiotic resistance in acute postoperative endophthalmitis. Ophthalmology 121: S1-9; quiz S10-12. [Crossref]

33. Miller D, Flynn PM, Scott IU, Alfonso EC, Flynn HW, Jr (2006) In vitro fluoroquinolone resistance in staphylococcal endophthalmitis isolates. Arch Ophthalmol 124: 479-483. [Crossref]

34. Stringham JD, Relhan N, Miller D, Flynn HW, Jr (2017) Trends in Fluoroquinolone Nonsusceptibility Among Coagulase-Negative Staphylococcus Isolates Causing Endophthalmitis, 1995-2016. JAMA Ophthalmol 135: 814-815. [Crossref]

35. Han DP1, Wisniewski SR, Wilson LA, Barza M, Vine AK, et al. (1996) Spectrum and susceptibilities of microbiologic isolates in the Endophthalmitis Vitrectomy Study. Am J Ophthalmol 122: 1-17. [Crossref]

36. Jones DB (2010) Emerging vancomycin resistance: what are we waiting for? Arch Ophthalmol 128: 789-791. [Crossref]

37. Das MK, Pathengay A, Shah GY, Koday NK (2011) Vancomycin-resistant coagulase negative Staphylococcus endophthalmitis following cataract surgery. J Cataract Refract Surg 37: 1908-1909. [Crossref]

38. Manav Khera, Avinash Pathengay, Animesh Jindal, Subhadra Jalali, Annie Mathai, et al. (2013) Vancomycin-resistant Gram-positive bacterial endophthalmitis: epidemiology, treatment options, and outcomes. J Ophthalmic Inflamm Infect 3: 46. [Crossref]

39. Witkin AJ, Chang DF, Jumper JM, Charles S, Eliott D, et al. (2017) VancomycinAssociated Hemorrhagic Occlusive Retinal Vasculitis: Clinical Characteristics of 36 Eyes. Ophthalmology. 124: 583-595. [Crossref]

40. Canadian Retina Society Executive B (2016) Canadian Retina Society ocular safety and therapeutics update: hemorrhagic occlusive retinal vasculitis. Can J Ophthalmol 51: 237. [Crossref]

41. Juárez-Verdayes MA, Reyes-López MA, Cancino-Díaz ME, Muñoz-Salas S, Rodríguez-Martínez S, et al. (2006) Isolation, vancomycin resistance and biofilm production of Staphylococcus epidermidis from patients with conjunctivitis, corneal ulcers, and endophthalmitis. Rev Latinoam Microbiol J 48: 238-246. [Crossref]

Copyright: (C2018 Velez-Montoya R. This is an open-access article distributed under the terms of the Creative Commons Attribution License, which permits unrestricted use, distribution, and reproduction in any medium, provided the original author and source are credited. 